\title{
Dekompressive Kraniektomie ohne Nutzen
}

Fragestellung: Ist die bilaterale dekompressive Kraniektomie bei Patienten mit erhöhtem Hirndruck als Folge eines SchädelHirn-Traumas (SHT) wirksam?

Hintergrund: Die wichtigste Komplikation des geschlossenen SHT ist die Entwicklung eines malignen Hirnödems, was in aller Regel durch eine medikamentöse Therapie nicht oder nur wenig beeinflusst werden kann. Zurzeit ist die dekompressive Kraniektomie die einzige Methode, mit der der Hirndruck bei diesen Patienten gesenkt werden kann. Dieses Therapiekonzept sollte jetzt in einer randomisierten Studie in den USA untersucht werden.

Patienten und Methodik: Die zwischen 2004 und 2014 durchgeführte Studie schloss Patienten im Alter zwischen zehn und 65 Jahren mit einem schweren SHT und einem therapierefraktär erhöhten intrakraniellen

Hutchinson PJ, Kolias AG, Timofeev IS et al. Trial of decompressive craniectomy for traumatic intracranial hypertension. N Engl J Med 2016; 375: 1119-30
Druck von mehr als 25 $\mathrm{mm}$ Hg ein. Die Patienten erhielten entweder eine bilaterale dekompressive Kraniektomie oder die konservative Therapie wurde fortgesetzt.
Der primäre Endpunkt war der Wert auf der „Extended Glasgow Outcome Scale“, einer 8-Punkte-Skala, die von Tod bis zu einem guten Outcome reicht. Die Auswertung erfolgte nach sechs Monaten.

Ergebnisse: Die Studie rekrutierte 408 Patienten, das mittlere Alter betrug 32 Jahre, $80 \%$ waren Männer. Etwa die Hälfte der Patienten hatten bei der Aufnahme einen Glasgow-Coma-Skala-Wert von 1 oder 2, die übrigen von 3 bis 6 . Etwa $30 \%$ der Patienten hatten eine Vorgeschichte mit Drogen- oder Alkoholmissbrauch. Bei $80 \%$ der Patienten lag ein diffuses Hirnödem vor. Über die einzelnen Kategorien der Extended-Glasgow-Outcome-Scale zeigte sich eine Reduktion der Sterblichkeit, aber eine deutliche Zunahme der Patienten, die in einem apallischen Syndrom verblieben. So betrug die Sterblichkeit $27 \%$ in der chirurgischen Therapiegruppe, verglichen mit $49 \%$ in der konservativen Behandlungsgruppe. Der Prozentsatz der Patienten mit einem schlechten funktionellen Outcome war in der chirurgischen Gruppe deutlich höher als in der konservativen Gruppe.

Schlussfolgerungen: Die dekompressive Kraniektomie bei Patienten mit erhöhtem Hirndruck nach SHT reduziert zwar die Mortalität, verschlechtert aber den funktionellen Outcome.

\section{- Kommentar von Hans-Christoph Diener, Essen}

\section{Ein ethisches Dilemma, das im Einzelfall diskutiert werden muss}

Die Ergebnisse dieser Studie sind ernüchternd. Im Gegensatz zu den Erwartungen führt eine bilaterale Hemikraniektomie beim malignen Hirnödem nach einem SHT nicht zu einer Reduktion der Zahl der schwerbehinderten Patienten. Die Sterblichkeit ist verringert, aber das wird durch einen hohen Prozentsatz von schwerstbehinderten Patienten erkauft. Hier besteht das Dilemma, ob es gerechtfertigt ist, durch einen operativen Eingriff die Sterblichkeit zu reduzieren, wenn gleichzeitig die Zahl der permanent pflegebedürftigen Patienten signifikant erhöht wird. Letztlich ist dies ein ethisches Dilemma, was im Einzelfall mit den Angehörigen der entsprechenden Patienten ausführlich diskutiert werden muss.

\section{Kombination mit einem Statin lohnt sich}

Fragestellung: Die Studie verglich den Outcome von Patienten mit Depression bei Anwendung eines selektiven Serotonin-Wiederaufnahmehemmers (SSRI) in Kombination mit Statinen versus der alleinigen Therapie mit SSRI.

Hintergrund: Aufgrund eines lediglich moderaten Ansprechens der antidepressiven Psychopharmakotherapie wurde die Suche nach möglichen Alternativen in zahlreichen Studien fokussiert. Hierbei erschien die Therapie mit antiinflammatorischen Substanzen relativ vielversprechend. In klinischen Studien konnte gezeigt werden, dass entzündungshemmende Medikamente wie selektive COX-2-Hemmer und Acetylsalicylsäure (ASS) positive Auswirkungen auf die depressive Symptomatik haben. Unabhängig vom lipidsenkenden Effekt konnte das auch bei Statinen beobachtet werden, obwohl verschiedene Untersuchungen teils gegensätzliche Ergebnisse lieferten. Dies bewog die Autoren der Studie eigene Recherchen zu dem Einsatz von Statinen in der Behandlung der Depression durchzuführen. Erste Tests im Tierversuch und beim Menschen suggerierten mögliche Vorteile beim kombinierten Einsatz von SSRI und Statinen im Ver- 